\title{
Further Comments on Models of Pathogen Evolution in Cultivar Mixtures and the Definition of Pathogen Fitness
}

\author{
Michael W. Shaw and Hanne Østergård
}

First author: School of Plant Sciences, The University of Reading, 2 Earley Gate, Whiteknights, Reading RG6 6AU, England; and second author: Plant Biology and Biogeochemistry Department, Ris $\emptyset$ National Laboratory, P.O. Box 49, DK, 4000 Roskilde, Denmark.

Accepted for publication 24 February 1998.

Yang and Ferrandino (12) make a number of thought-provoking points about Østergård and Shaw (8) and Sun and Zeng (11) that we would like to comment on. We first clarify our concern about the estimation procedure proposed in Sun and Zeng (11) and then respond to some specific comments made in Yang and Ferrandino (12).

Changes in frequencies of pathogen genotypes in cultivar mixtures or other heterogeneous host populations have been modeled for at least two reasons: (i) to predict theoretically how genotype distributions will evolve $(1,6,7)$; and (ii) to give an expected pattern or null hypothesis with which to compare observed changes (5). Sun and Zeng (11) proposed a method to estimate relative fitnesses of pathogen genotypes growing on a heterogeneous host using observations of changes in frequencies of the genotypes on several mixtures of different composition. Sun and Zeng (11) suggested that their method could be used also to predict the effect of host composition on "race" frequencies. Their method was based on writing the change in frequencies of one genotype relative to a reference genotype as a linear combination of parameters, $f_{i j k}$, with the frequencies of the components as the coefficients (11). They described $f_{i j k}$ as "the relative seasonal fitness of race $i$ to race $j$ on cultivar $k$." Østergård and Shaw (8) used a deliberately very simple situation to show that the relative changes in genotype frequencies in a mixture cannot, in general, be written as a linear function of the relative fitnesses on the pure components with the frequencies of the components as the coefficients. If the purpose of Sun and Zeng's (11) method is to relate the observed changes of genotype frequencies in mixtures to the proportions of cultivars used in various experiments, the regression coefficients $f_{i j k}$ cannot be interpreted as relative fitnesses on the components as Sun and Zeng (11) define them in their Equation 2. A simple example will clarify this.

Consider two cultivars, A and B, and two pathogen genotypes, 1 and 2. Over a season, the genotypes produce offspring per original spore on isolated plots of each cultivar as implied in Table 1, lines 1 and 2. Thus, following Sun and Zeng (11), the relative fitnesses are to be referred to genotype 1, because it has the greatest absolute fitness on any cultivar in the system. The fitness of genotype 2 relative to genotype 1 on cultivar $\mathrm{A}$ is $3 / 4$, and on cultivar 2 , it is 0 . Now suppose Sun and Zeng's (11) procedure is followed in a very small experiment, using just a 0.5:0.5 mixture and a 0.8:0.2 mixture (Table 1, lines 3 and 4). For simplicity only, we assume there is no spread of inoculum between plants. We further assume that the initial inoculum is evenly spread over the mixture components, so that the initial density is the same on each cultivar. At the end of the season in the 0.5:0.5 mixture, twice as many spores of genotype 1 as of genotype 2 have been produced per initial spore, so the relative fitness of genotype 2 to genotype 1 is $1 / 2$. In

Corresponding author: M. W. Shaw; E-mail address: M.W.Shaw@reading.ac.uk

Publication no. P-1998-0325-030

(C) 1998 The American Phytopathological Society the 0.8:0.2 mixture this value is $2 / 3$. Now, these figures can be inserted into the least squares equations suggested by Sun and Zeng (11), which will, in this case, have an exact solution since there are only two equations in two unknowns. Since there is no error, any estimation procedure should recover the original relative fitnesses. We denote estimated quantities by $\hat{f}_{i j k}$ and the corresponding observed ones by $f_{i j k}$. After solving the equations, we obtain $\hat{f}_{21 \mathrm{~A}}={ }^{7} / 9$ and $\hat{f}_{21 \mathrm{~B}}=2 / 9$. But, in the construction of the example, $f_{21 \mathrm{~A}}=3 / 4$ and $f_{21 \mathrm{~B}}=0$ ! In conclusion, the procedure is not consistent. Furthermore, from experiments using different sets of mixtures, a very wide range of estimates, possibly including negative values, can be obtained even though there is no experimental error and the biology of the host-pathogen interaction is unchanged. In general, the regression coefficients will be strongly dependent on the exact set of mixture compositions used in the estimating equations, and the fit of the equations will not necessarily be good.

We would next like to comment on the second part of Yang and Ferrandino's (12) paper, in which they present definitions of fitness that they feel will better correspond to the intuitive notion. Given the confusion, this seems a good idea! (Yang and Ferrandino [12] use the phrase "population numbers" on each cultivar. We take this to mean population densities, since if actual population numbers are used, the cultivar density in effect enters twice into their Equation 6.) Yang and Ferrandino's (12) Equation 9 is, as they point out, a restatement of the basic comparison of frequencies made in their Equation 1 (which is Equation 1 in Østergård and Shaw [8] and Equation 2 in Sun and Zeng [11]). We would definitely agree that this ratio, Yang and Ferrandino's (12) Equation 9 , is a measurement of the relative fitness of two isolates on a given mixture or pure cultivar, and that this definition is fundamental. We would go further and say that it is basically the same definition used by both Østergård and Shaw (8) and Sun and Zeng (11). However, the definition by itself does not allow measurements of relative fitness made on single cultivars to be related to measurements of relative fitness on mixtures. Sun and Zeng (11) proposed to predict changes in frequencies in single cultivars and arbitrary mixtures using experimental data on changes in frequencies in a restricted set of mixtures. Yang and Ferrandino (12) do not attempt this task. The derivation in Østergård and Shaw (8) was not intended as a general method to make this connection nor as an introduction of a new set of definitions, as was made clear in that paper. We would emphasize that the aim in presenting Equations 5 to 9 in Østergård and Shaw (8) was to set out the problems demonstrated above that we felt Sun and Zeng's (11) procedure for estimating fitnesses on mixture components raised. We chose a drastically simplified (and therefore unrealistic) situation, since any suggested general method should at least be logically consistent in such a case. Yang and Ferrandino (12) point out that their Equation 6 differs in general from Østergård and Shaw's (8) Equation 6. The two equations are the same if the initial population densities for a genotype, $P_{i k 0}$, are the same on each component of 
TABLE 1. Fitnesses and spore densities at the end of the season for two genotypes on two hypothetical host cultivars and two different mixtures of those cultivars ${ }^{\mathrm{a}}$

\begin{tabular}{|c|c|c|c|}
\hline & \multicolumn{2}{|c|}{ Spore density } & \multirow{2}{*}{$\begin{array}{l}\text { Fitness of genotype } 2 \\
\text { relative to genotype } 1^{b}\end{array}$} \\
\hline & Genotype 1 & Genotype 2 & \\
\hline Cultivar A & 200 & 150 & $3 / 4$ \\
\hline Cultivar B & 100 & 0 & 0 \\
\hline $0.5 \mathrm{~A}+0.5 \mathrm{~B}$ & 150 & 75 & $1 / 2$ \\
\hline $0.8 \mathrm{~A}+0.2 \mathrm{~B}$ & 180 & 120 & $2 / 3$ \\
\hline
\end{tabular}

a The two genotypes are of a pathogen initially inoculated uniformly at a density of 1 per unit area. It is assumed there is no spread of inoculum between plants.

b

$$
\left[\left(\sum_{k} c_{k} P_{2 k}\right) /\left(\sum_{k} c_{k} P_{2 k 0}\right)\right] /\left[\left(\sum_{k} c_{k} P_{1 k}\right) /\left(\sum_{k} c_{k} P_{1 k 0}\right)\right]
$$

using the notation of Yang and Ferrandino's (12) Equation 9. $P_{i k}$ represents spore density; $P_{i k 0}$ represents initial spore density. $P_{i k 0}=1$ for all $i$ and $k$. $c_{k}$ represents the proportion of cultivar $k$ in the mixture. $i \in\{1,2\} ; k \in\{\mathrm{A}, \mathrm{B}\}$. The spore density columns are the denominator and numerator of the formula.

the mixture. This assumption was not explicit in Østergård and Shaw (8), because we had in mind a cultivar mixture with plants intimately mixed and the population measured by estimating spore density; we apologize for any confusion.

Third, we would like to discuss the range in which relative fitness values can lie. Yang and Ferrandino (12) suggest in the first part of their paper that our (8) criticism of Sun and Zeng (11) is invalid, because we used fitnesses in the range 0 to $\infty$, whereas Sun and Zeng (11) had suggested that the fitnesses on mixture components should be arranged to lie between 0 and 1. Yang and Ferrandino (12) argue that this is possible, in the lines following their Equation 2. They also point out that their Equation 9 allows all relative fitnesses on a particular mixture to be in the range $(0,1)$ by choosing as reference genotype the genotype for which their Equation 6 is maximal. However, if the values of $c_{k}$ (proportion of cultivar $k$ in the mixture) were changed, or an extra genotype introduced, a new reference genotype would have to be selected, which might be different. It would then be impossible to compare relative fitnesses across mixtures of different compositions. If only one cultivar is considered, fitness can indeed always be expressed relative to the most fit genotype, as in Yang and Ferrandino's (12) references 1 to 4 . However, as soon as more than one cultivar or mixture is considered, there need be no choice of reference genotype that makes it possible to have all fitnesses less than 1. This is because a different isolate may be the most fit on different cultivars. In consequence, the most fit on a mixture may depend on the composition of the mixture. Table 2 sets out an example showing this. Defining fitnesses relative to a constant reference genotype to be less than 1 corresponds to a genuine and quite severe restriction on the biological systems that can be considered, for little mathematical gain. In evolutionary problems concerning different environments, relative fitnesses must, in general, be between 0 and $\infty$ inclusive $(2,3,4,9,10)$. In fact, none of the papers cited by Yang and Ferrandino (12) discuss both multiple pathogen genotypes and multiple hosts, so the situation did not arise for those authors.

In the special case in which the most fit genotype on any host was also the most fit on all other hosts, all fitnesses could be less than or equal to 1. Even then, however, the procedure in Sun and Zeng (11) unfortunately gives results that will generally be logically inconsistent, as shown by the calculations based on Table 1.
TABLE 2. Fitnesses and spore densities at the end of the season for two genotypes on two hypothetical host cultivars and two different mixtures of those cultivars ${ }^{\mathrm{a}}$

\begin{tabular}{|c|c|c|c|c|c|}
\hline & \multicolumn{2}{|c|}{ Spore densities } & \multirow[b]{2}{*}{$\begin{array}{l}\text { Most fit } \\
\text { genotype }\end{array}$} & \multicolumn{2}{|c|}{ Fitness $^{\mathrm{b}}$ of genotype } \\
\hline & $\begin{array}{c}\text { Genotype } \\
1\end{array}$ & $\begin{array}{c}\text { Genotype } \\
2\end{array}$ & & $\begin{array}{l}1 \text { relative } \\
\text { to } 2\end{array}$ & $\begin{array}{c}2 \text { relative } \\
\text { to } 1\end{array}$ \\
\hline Cultivar C & 200 & 100 & 1 & 2 & 0.5 \\
\hline Cultivar D & 100 & 400 & 2 & 0.25 & 4 \\
\hline $0.2 \mathrm{C}+0.8 \mathrm{D}$ & 120 & 340 & 2 & 0.35 & 2.83 \\
\hline $0.8 \mathrm{C}+0.2 \mathrm{D}$ & 180 & 160 & 1 & 1.13 & 0.89 \\
\hline
\end{tabular}

a The two genotypes are of a pathogen initially inoculated uniformly at a density of 1 per unit area. It is assumed there is no spread of inoculum between plants.

$$
\left[\left(\sum_{k} c_{k} P_{2 k}\right) /\left(\sum_{k} c_{k} P_{2 k 0}\right)\right] /\left[\left(\sum_{k} c_{k} P_{1 k}\right) /\left(\sum_{k} c_{k} P_{1 k 0}\right)\right]
$$

using the notation of Yang and Ferrandino's (12) Equation 9. $P_{i k}$ represents spore density; $P_{i k 0}$ represents initial spore density. $P_{i k 0}=1$ for all $i$ and $k$. $c_{k}$ represents the proportion of cultivar $k$ in the mixture. $i, j \in\{1,2\} ; i \neq j ; k \in\{\mathrm{C}, \mathrm{D}\}$.

The fundamental problem is, as Yang and Ferrandino (12) correctly point out just after their Equation 5, that the functions relating population multiplication on one cultivar to multiplication on a mixture are nonlinear. Our (8) criticism of Sun and Zeng's (11) procedure depends on this fact and does not depend on the range within which it is necessary to define the individual relative fitnesses, though cases in which different genotypes are most fit on different cultivars produce the most dramatic counterexamples.

In conclusion, we hope that all the authors in this series of papers would agree on at least two things: the importance of studying the evolution of virulence in heterogeneous environments, and a commitment to logical clarity. We hope that our joint contributions have served these ends.

\section{LITERATURE CITED}

1. Barrett, J. A. 1978. A model of epidemic development in variety mixtures. Pages 129-137 in: Plant Disease Epidemiology, P. R. Scott and A. Bainbridge, eds. Blackwell Scientific Publications, Oxford.

2. Barton, N., and Clark, A. 1990. Population structure and processes in evolution. Pages 115-173 in: Population Biology. K. Wöhrmann and S. K. Jain, eds. Springer-Verlag, Berlin.

3. Barton, N. H. 1979. Gene flow past a cline. Heredity 43:333-339.

4. Christiansen, F. B. 1990. Natural selection: Measures and modes. Pages 27-81 in: Population Biology. K. Wöhrmann and S. K. Jain, eds. Springer Verlag, Berlin.

5. Hovmøller, M. S., Munk, L., and Østergård, H. 1993. Observed and predicted changes in virulence gene frequencies at 11 loci in a local barley powdery mildew population. Phytopathology 83:253-260.

6. Lannou, C., and Mundt, C. C. 1996. Evolution of a pathogen population in host mixtures-Simple race-complex race competition. Plant Pathol. 45:440-453.

7. Østergård, H. 1983. Predicting development of epidemics on cultivar mixtures. Phytopathology 73:166-172.

8. Østergård, H., and Shaw, M. W. 1996. Linear models are inappropriate to estimate relative parasitic fitness of pathogens in heterogeneous host populations. Phytopathology 86:561-562.

9. Slatkin, M. 1973. Gene flow and selection in a cline. Genetics 75:733-756.

10. Strickberger, M. W. 1968. Genetics. Macmillan, New York.

11. Sun, P., and Zeng, S. 1995. Estimation of relative parasitic fitness of pathogens in heterogeneous host populations. Phytopathology 85:520-521.

12. Yang, X. B., and Ferrandino, F. J. 1998. Linear models to estimate relative fitness: A reply. Phytopathology 88:490-491. 